\title{
Modeling of Broadband Liners Applied to the Advanced Noise Control Fan
}

\author{
Douglas M. Nark* and Michael G. Jones ${ }^{\dagger}$ \\ NASA Langley Research Center, Hampton, VA 23681-2199 \\ Daniel L. Sutliff ${ }^{\ddagger}$ \\ NASA Glenn Research Center, Cleveland, OH 44212
}

\begin{abstract}
The broadband component of fan noise has grown in relevance with an increase in bypass ratio and incorporation of advanced fan designs. Therefore, while the attenuation of fan tones remains a major factor in engine nacelle acoustic liner design, the simultaneous reduction of broadband fan noise levels has received increased interest. As such, a previous investigation focused on improvements to an established broadband acoustic liner optimization process using the Advanced Noise Control Fan (ANCF) rig as a demonstrator. Constant-depth, double-degree of freedom and variable-depth, multi-degree of freedom liner designs were carried through design, fabrication, and testing. This paper addresses a number of areas for further research identified in the initial assessment of the ANCF study. Specifically, incident source specification and uncertainty in some aspects of the predicted liner impedances are addressed. This information is incorporated in updated predictions of the liner performance and comparisons with measurement are greatly improved. Results illustrate the value of the design process in concurrently evaluating the relative costs/benefits of various liner designs. This study also provides further confidence in the integrated use of duct acoustic propagation/radiation and liner modeling tools in the design and evaluation of novel broadband liner concepts for complex engine configurations.
\end{abstract}

\section{Nomenclature}

Symbols:

sound speed

liner core depth

annular duct height (outer radius - inner radius)

free space wave number

axial liner length

circumferential mode number

\footnotetext{
${ }^{*}$ Research Scientist, Structural Acoustics Branch, Research Directorate, AIAA Associate Fellow

${ }^{\dagger}$ Senior Research Scientist, Structural Acoustics Branch, Research Directorate, AIAA Associate Fellow

${ }^{\ddagger}$ Aerospace Engineer, Acoustics Branch, AIAA Associate Fellow
} 


\section{Introduction}

As modern turbofan engines have incorporated increased bypass ratios and advanced fan designs, the broadband component of fan noise has grown in relevance. Therefore, while the attenuation of fan tones remains a major factor in engine nacelle acoustic liner design, the simultaneous reduction of broadband fan noise levels has received increased interest. As such, a previous investigation focused on improvements to an established broadband acoustic liner optimization process using the Advanced Noise Control Fan (ANCF) rig as a demonstrator ${ }^{112}$ Specifically, induct attenuation predictions with a statistical source model were used to obtain optimum impedance spectra over the conditions of interest. The predicted optimum impedance information was then combined with liner modeling tools to design liners that most closely matched the predicted optimum values. Design selection was based on an acceptance criterion that provided the ability to apply increased weighting to specific frequencies and/or operating conditions. Constant-depth, double-degree of freedom and variable-depth, multi-degree of freedom designs were carried through design, fabrication, and testing. Predicted and measured liner performance (in-duct attenuation) was then compared to validate the efficacy of the design process.

Through the course of the previous study, a number of opportunities for further research were identified. First, the difference in source specification between predictions and measurements was identified as a possible source of discrepancy in the associated attenuation values. Additionally, the need for certain test hardware components led to uncertainty in the as-built impedances for both test liners. Specifically, this included the effects of compliance of the centerbody material (considered rigid in the predictions), as well as the effects of a retaining screen required for the liner spool assembly. Subsequently, information on the actual source content measured with a rotating rake became available and testing was performed to reduce the uncertainty in the liner impedances. The remainder of the paper details the updated source information and test results, as well as the resultant predictions of the broadband liner designs. The selected test configuration and operating conditions of interest are presented in Section III This is followed by a discussion of the computational approach and implementation in Section III] and the liner modeling in Section IV. Comparison of predicted and measured results and evaluation of liner performance are then presented in Section $\mathrm{V}$ Finally, concluding remarks regarding some of the more significant results and further areas of interest are presented in Section $\mathrm{VI}$

\section{Test Configuration}

The test bed selected for this study was the Advanced Noise Control Fan (ANCF),,$\frac{3,45}{3}$ a 4-foot diameter low speed fan used for validation of noise reduction concepts. The ANCF, shown in Figure 1a operates inside an enclosed anechoic dome of the Aero-Acoustic Propulsion Laboratory at NASA Glenn Research Center. The nominal operating condition of the ANCF is 1800 RPM (tip speed $375 \mathrm{ft} / \mathrm{sec}$ ) providing an inlet duct Mach number of $\sim 0.15$ and a fundamental blade passing frequency (BPF) of $\sim 500 \mathrm{~Hz}$. The ANCF is comprised of a series of 11 and 12 inch long cylindrical spools that are axially interchangeable, enabling rapid testing of a variety of configurations. As an alternative to the fan source, the Configurable Fan Artificial Noise System (CFANS) may be utilized to generate and control circumferential modal source content. The system consists of four rings of 16 circumferentially distributed sets of electromagnetic drivers flush-mounted in the inner wall at four axial locations. The ANCF also allows for the insertion of a rotating microphone rake to measure modal content ${ }^{[6]}$ Therefore, for selected test configurations, in-duct rotating rake measurements and farfield acoustics measurements may be obtained.

Further discussion of the ANCF and full test program may be found in a companion pape ${ }^{[2}$ to the initial study. However, this work focuses on the liner evaluation process for a non-standard configuration used for initial testing. Prior to studying the standard horizontal configuration, testing was conducted with the ANCF hardware in a vertical orientation to provide a clean, annular duct for experimentation. In this case, the spool pieces were stacked up in a vertical orientation on the floor as shown in Figure $1 \mathrm{~b}$. This removed the support hardware of the standard arrangement, providing the basis for a constant area annular duct. Two such configurations were achieved through the use of two cylindrical centerbodies with constant diameters of $24 \mathrm{in} .(60.96 \mathrm{~cm})$ and $36 \mathrm{in}$. $(91.44 \mathrm{~cm})$. These correspond to equivalent annular duct hub-to-tip ratios, $\tau$, of 0.5 and 0.75 , respectively. The entire stack rested on the floor, and approximately 6 in. $(15.24 \mathrm{~cm})$ of foam material was placed in the bottom of the stack to minimize reflections from the floor. For this no-flow configuration, in-duct rotating rake measurements were acquired at the entrance and exit of the lined section to determine attenuation due to the acoustic liner. The CFANS was used to generate individual circumferential modes of order, $m$, ranging from 0 to 6 over a frequency range of 500 to $1500 \mathrm{~Hz}$. In addition, a source field was generated that consisted of all possible modes with a table of randomized phase values. These phases were set in a deterministic manner (i.e., the same set of random phases was used for each test). In contrast to the 
specification of individual circumferential modes, this latter source more closely approximates the fully randomized source used in the liner design process (see Section IIII). However, comparison of measured and predicted attenuation values for both source specifications are presented in Section $\mathrm{V}$

\section{Numerical Approach and Implementation}

The CDUCT-LaRC (CDL) duct propagation and radiation code was used to obtain predicted attenuation (difference in acoustic power between the exhaust and source planes) values. This code calculates the propagation of a given acoustic source ahead of the fan face or aft of the exhaust guide vanes in the inlet or exhaust ducts, respectively. The three-dimensional duct may be acoustically lined (possibly circumferentially and radially segmented) in specified areas and incorporate struts/bifurcations. All of the modules that currently make up the CDL framework have been discussed previously ${ }^{7}$ However, this discussion focuses on the propagation module, as it is most pertinent to this investigation.

The duct propagation module is based on the CDUCT code developed by Dougherty ${ }^{89}$ and extended by Lan.10 This code utilizes a parabolic approximation to the convected Helmholtz equation and offers a computationally efficient model that accounts for the complexities of fully three-dimensional nacelle configurations. The CDL code has been extended ${ }^{77}$ to support multi-block propagation calculations. The grid connectivity is determined and data is transferred from upstream to downstream blocks without user intervention. The output of the propagation module includes the acoustic potential or pressure within the duct, which may be utilized by the radiation module for acoustic radiation calculations.

While CDL can accept arbitrary source specification (i.e., it is not a modal code), it is convenient to specify the acoustic source distribution in terms of duct modes. For situations in which the source pressure is available, this greatly simplifies the conversion to the required acoustic potential. However, when source information is not available, an assumption on the source description must be made. This is generally the case and the approach taken here follows that described by Zlavog and Eversman ${ }^{11 \mid 12}$ in a series of statistical studies into the effects of randomized modal source power and/or phase on attenuation in lined ducts. In this study, the source modal powers (and hence, amplitudes) and modal phases were allowed to vary randomly and independently. Thus, with equal probability of occurrence, the amplitude and phase for each cut-on source mode may take any value in the intervals from 0 to 1 and 0 to $2 \pi$, respectively. This uncertainty in source specification results in uncertainty in the predicted sound levels. However, as discussed by Zlavog and Eversman, ${ }^{11 / 12}$ for the case of all propagating circumferential and radial acoustic modes with random modal power and phase, transmitted power appears to be normally distributed. In their statistical studies, this also produced statistical distributions with the least standard deviation. Therefore, as discussed in a previous CDL study ${ }^{\sqrt{13}}$ Student's t-distribution was used to statistically analyze the predicted attenuation results. For each configuration, 11 simulations were performed to produce a sample population from which mean, $\mu$, and standard deviation, $\sigma$, values can be calculated.

Using in-duct attenuation as the cost function, optimum impedance predictions were obtained for frequencies ranging from $500 \mathrm{~Hz}$ to $1500 \mathrm{~Hz}$ in $100 \mathrm{~Hz}$ increments with zero mean flow. Initial calculations focused on the $\tau=0.5$ vertical configuration and showed that only modest attenuation could be achieved at the given hub-to-tip ratio. As shown by $\mathrm{Kraft}^{14}$ and Rice, $\frac{15}{15}$ this is to be expected due to the ratio of liner axial length to duct height $(L / H)$ and frequencies of interest. While this provided data for further validation of the broadband liner design process, configurations allowing for larger liner benefits were desired. Therefore, based on further predictions and the versatility of the vertical test set-up, the $\tau=0.75$ case was included in testing. This provided additional validation data, as well as a configuration for which larger liner attenuation could be achieved. Ultimately, the predicted optimum impedance for the $\tau=0.5$ case was selected as the design target. This was mainly due to the fact that the constant area section of the standard ANCF configuration (on stanchion, see Fig 1al) has a 24 inch centerbody. However, the difference between the predicted optimum impedance spectra for the two cases was also minimal.

\section{Liner Modeling and Fabrication}

Both a constant-depth, double-degree of freedom (DDOF) and a variable-depth, multi-degree of freedom (MDOF) liner were designed and manufactured. To achieve broadband performance, the MDOF liner consisted of a multilayer configuration incorporating septa (or "mesh-caps") embedded into a honeycomb core. This concept allows the acoustic liner to be customized such that the surface impedance of each individual cell is independently varied. The various cells are then arranged into a grid pattern of different mesh-cap depths or resistances within the acoustic panel to achieve a desired distributed impedance. 
Due to the relatively low target optimum resistance values, a $0.05 \rho c$ wiremesh facesheet was selected for both liners. This minimized the impact of the facesheet on the overall resistance and allowed for greater flexibility in tuning the resistance of individual cells via the embedded mesh-caps. The liner core was comprised of a 0.375 -inch ( 0.953 $\mathrm{cm})$ diameter hexagonal-shaped honeycomb core with an overall depth of 2.0 inches $(5.08 \mathrm{~cm})$. Mesh-cap materials ranging in DC flow resistance values from 1.5 to $3.0 \rho c$ were considered. Additionally, the distance between the meshcap and liner backplate, $h_{1}$, was allowed to vary (see Figure 2). In the design process, this value ranged from 0.20 in $(0.508 \mathrm{~cm})$ to 1.80 in $(4.57 \mathrm{~cm})$ at 0.20 in $(0.508 \mathrm{~cm})$ increments. An optimization process was used to determine the best combination of geometrical parameters to achieve the desired impedance values. For the DDOF liner, the design was constrained to a single constant mesh cap resistance and insertion depth for all cells. Conversely, for the MDOF liner, a four-cell combination of mesh cap resistance and insertion depth was utilized.

The final core designs were incorporated into an existing spool piece having a 48 inch $(121.92 \mathrm{~cm})$ inner diameter, an effective axial length of 16 inches $(40.64 \mathrm{~cm})$, and approximately 4 inch $(10.16 \mathrm{~cm})$ depth. A photograph of the empty spool piece is shown in Figure 3a The liner assembly (see Figure 4 was built up starting from the outer radius and working inward. In order to provide a compression mechanism for the entire assembly, two layers of carpet padding were first laid in the spool. This accounted for geometric imperfections in the spool wall and allowed for a tight fit. A hard rubber sheet was then inserted to provide a seal at the base of the honeycomb core and prevent acoustic leakage between adjacent cells. One of the two liner cores was then placed on top of this rubber sheet, followed by the wire mesh facesheet (DC flow resistance of $0.05 \rho c$ ). Finally, a coarse (retaining) screen was fastened to the facing of the inner ledge of the spool frame to compress the entire lay-up securely and provide a flush flow surface in the duct. A photograph of an installed liner spool piece is shown in Figure 3b

The overall designs for both liners attempted to account for this assembly process. For example, the predicted compliance of the rubber backing was taken into account, rather than assuming a rigid backplate. However, as mentioned previously, there was some initial uncertainty in the modeling of the centerbody material (assumed to be rigid) and coarse screen. Follow up testing of the centerbody material demonstrated the rigid wall assumption to be appropriate. Consideration of the coarse screen was more problematic. At the time of design, the coarse screen was assumed to have negligible effect on the overall impedance. However, after core fabrication, it was estimated that the screen would increase resistance by as much as $0.5 \rho c$ (based on approximate open area) and predicted impedances were adjusted accordingly. It became clear from comparisons of predicted and measured in-duct attenuation that the actual screen impedance was between the two extremes and required further investigation. Therefore, testing of low-resistance wiremesh facesheets bonded to representative coarse screens was conducted to better quantify the interaction. Results showed that the addition of the coarse screen increased resistance by only $0.036 \rho c$ with negligible impact on the reactance (i.e. much closer to the initial assumption neglecting the effects of the screen on overall liner impedance). As such, the predicted impedances were once again adjusted to account for this updated information.

The DDOF and MDOF liners were tested in separate assemblies. To provide a larger measurement database, as well as assessment of the prediction methodology for segmented liners, aluminum tape was used to cover portions of the lined section and generate additional liner configurations. The end result was a fully hardwall (A) configuration, fully lined (B) configuration, and three segmented (C-E) configurations. Configurations B-E are illustrated in Figure 5 where the lined portions appear as red wireframe. The key dimensions are also provided in Table 1 with axial dimensions relative to the liner leading edge (LE). All five configurations of the DDOF and MDOF were tested with the 24 in. centerbody $(\tau=0.5)$. As for the 36 in. centerbody $(\tau=0.75)$, only the MDOF liner was tested (again with all five configurations).

The predicted impedances for both liners compared with the predicted optimum for the $\tau=0.5$ configuration are shown in Figures 6. It can be seen that the character of the target impedance (relatively low resistance at lower frequencies and decrease in reactance with increasing frequency) make it difficult to match with a physically-realizable liner. Even with a low resistance facesheet, the design resistance values are above the optimum value at the lower frequencies. Additionally, the $-\cot (k d)$ behavior evident in the design reactance spectra is counter to the optimum reactance trend. The predicted mean attenuation associated with the optimum $(\tau=0.5)$, DDOF, and MDOF impedance spectra are shown in Figures $7 \mathrm{a}$ and $7 \mathrm{~b}$, respectively. Due to the statistical nature of the source, error bars representing the $95 \%$ confidence intervals for the mean attenuation are also included in these figures. The issues with low achievable attenuation discussed previously are visible in Figure $7 \mathrm{a}$, although the upper bounds of the $95 \%$ confidence intervals indicate the possibility of attenuation values close to $6 \mathrm{~dB}$ at some frequencies. The difficulty in matching the optimum impedance at the low frequencies is also apparent, as the predicted attenuation values for the DDOF and MDOF liners are below those predicted for the optimum. The increased attenuation resulting from the larger centerbody (and hence $L / H)$ are evident in Figure $7 \mathrm{~b}$. This is an example of a case in which the possible advantage of the MDOF approach may not be realized for the given cost function. In this situation, the ability to achieve a small increase in attenuation 
at higher frequencies with an associated larger decrease in attenuation at the lower frequencies is probably not cost effective. However, as the various liner designs (i.e., DDOF, MDOF, etc.) and their relative costs/benefits may be assessed concurrently, this illustrates the value of the design process. It should also be noted that demonstrating large attenuation in the ANCF was not a goal of this study, but rather validating the efficacy of the liner design process via comparison of the predicted performance with measured results.

\section{Results and Evaluation}

In order to evaluate the liner performance and provide further confidence in the liner design process, comparisons between predicted and measured attenuation are performed. The measured values represent insertion loss obtained from the aforementioned rotating rake data which, based on previous experience, are assumed to have a measurement error of $+/-1 \mathrm{~dB} ?^{[2]}$ The predicted attenuation values are obtained using the CDL code (see Section III) and, when appropriate, error bars represent the estimated $95 \%$ confidence intervals for the mean attenuation. Beginning with cases consisting of individual circumferential mode sources, comparison of measured and predicted attenuations for the MDOF liner are presented in Figure 8 Here, the CFANS are driven in such a way that the modal power is spread across the cut-on radial modes of a selected circumferential mode number (shown on the abscissa). For the frequency $(f=1100 \mathrm{~Hz})$ and centerbody $(\tau=0.75)$ considered, conditions are such that only a single radial mode is cut-on for a given circumferential mode number. Comparisons between measured and predicted attenuations are very good across the liner configurations with a slightly larger deviation for configuration C (Fig. 8a). However, even in this case, the comparisons would appear to be within expected uncertainties.

For the smaller centerbody $(\tau=0.5)$ and same frequency $(f=1100 \mathrm{~Hz})$, the modal source is slightly more complicated. In this situation, conditions are such that more than one radial mode is cut-on for a given circumferential mode. As pointed out by Zlavog and Eversman, $\frac{16}{16}$ for cases of multiple mode input, optimum impedance and realized attenuation depend strongly on modal phases. This influence of modal phase variation on attenuation decreases as the number of cut-on (i.e., propagating) modes increases. Therefore, as the predictions use rotating rake data to specify the incident sound field, it is expected that predicted attenuations would be sensitive to relative phase errors. This is illustrated in Figure 9 in which the measured and predicted attenuation comparisons are presented in a slightly different format. Here, the predicted attenuation for the MDOF liner obtained from direct rotating rake data is shown as an open symbol. As expected, based on the previous discussion of modal content, there is a larger disparity between these and the measured values. To investigate the effect of modal phase, predictions were obtained for which the modal source amplitudes were based on rotating rake data, but the modal phases were randomized. When only phase is randomized for a given circumferential mode, the transmitted power is generally not normally distributed (Gaussian). ${ }^{11}$ However, for the illustrative purposes of this study, it is assumed to be sufficiently close to Gaussian to use the same processing. Therefore, as with the statistical source processing in Section III 11 simulations were performed for each circumferential mode to produce a sample population from which mean, $\mu$, and standard deviation, $\sigma$, values can be calculated. The results are presented in Figures 9 using closed symbols to represent the mean attenuation values and error bars to represent the "estimated" $95 \%$ confidence intervals. It can be seen that the measured attenuation values fall within the predicted confidence intervals (with a few exceptions) and the measured trends are captured in the predicted mean attenuation values. To further illustrate this behavior, results for the larger centerbody $(\tau=0.75)$ at a higher frequency $(f=1500 \mathrm{~Hz})$ are shown in Figure $9 \mathrm{~d}$ Except for an anomaly at $m=1$ a the measured attenuation values again fall within the predicted confidence intervals and the measured trends are captured in the predicted mean attenuation values. The results provided in Figures 8 - 9 are illustrative of comparisons across all configurations involving individual circumferential mode sources. Predicted and measured attenuation values compare very well for single cut-on radial source modes (Fig. 8). For cases involving more than one cut-on radial source mode (Fig. 9), the measured attenuation values generally fall within the predicted confidence intervals and the measured trends are well captured by the predicted mean attenuation values.

In addition to the individual circumferential mode source cases, those involving all cut-on source modes were investigated. These include configuration B of the DDOF and MDOF liners with the small centerbody $(\tau=0.5)$, as well as configuration B of the MDOF liner with the large centerbody $(\tau=0.75)$. Measured attenuation values were obtained over a frequency range from $500 \mathrm{~Hz}$ to $1500 \mathrm{~Hz}$ in $200 \mathrm{~Hz}$ increments. Predictions were initially performed with the full statistical source (random source mode amplitude and phase) used in the optimization. These were followed by predictions using the rotating rake data for source specification. As with the individual circumferential

\footnotetext{
${ }^{\text {a }}$ The rotating rake data also showed considerable power in the $m=-15$ circumferential mode, which is very close to cut-off at the given conditions. With slight differences in ambient conditions leading to cut-on/cut-off, the presence (or absence) of this mode would appear to be a strong candidate for the apparent anomalous behavior.
} 
mode cases having multiple cut-on radial modes, these included both direct rotating rake information and fixed modal amplitude with randomized phases.

Comparison of measured and predicted attenuations for the DDOF and MDOF liners for the small centerbody are presented in Figures 10-11. The prediction of lower achievable attenuations for the $\tau=0.5$ case is confirmed in the measured data. However, predictions using the full statistical source under-predict the measured values at several frequencies, particularly 700 and $1300 \mathrm{~Hz}$. Use of the rotating rake source information slightly increases the predicted mean attenuation values and provides comparisons that nearly fall within expected uncertainties. Results for the MDOF liner for the $\tau=0.75$ case are provided in Figure 12 Comparison between predicted and measured values are very good for both source specification $\$$ It is interesting to note that the location of maximum attenuation observed in both the predictions and measurements corresponds closely with the frequency $(\sim 1100 \mathrm{~Hz})$ at which the design and optimum reactance values are most closely matched (see Figure 6). Perhaps more importantly, the findings also provide further evidence to support the expectation that the CDL code can be used to investigate various liner configurations and provide conservative attenuation predictions that correctly capture measured trends.

\section{Concluding Remarks}

This paper describes improvements to a previously established process for the design of novel broadband acoustic liner concepts with limited fan source information. The selection of the Advanced Noise Control Fan (ANCF) rig afforded the opportunity to include liner fabrication and testing to further validate the broadband liner design process. Predictions on the initial test configuration indicated an optimum impedance spectrum that was difficult to achieve, accompanied by relatively low achievable attenuation values. However, the versatility of the vertical test set-up allowed for an additional configuration, for which larger attenuation values could be achieved, to be included in the test matrix. Early stages of the design process also showed the ANCF to represent a case in which the possible advantage of the multi-degree of freedom (MDOF) approach may not be realized. In comparison with the double-degree of freedom (DDOF) design, the MDOF design showed a small increase in attenuation at higher frequencies with an associated larger decrease in attenuation at the lower frequencies that may not be cost effective. This is, of course, dependent on the chosen cost function and configuration, but illustrated the value of the design process in concurrently evaluating various liner designs (i.e., DDOF, MDOF, etc.) and their relative costs/benefits. In addition, a main goal of validating the efficacy of the design process through design, fabrication, and evaluation of various liner designs was met.

As identified in a previous study, 1 a number of areas requiring attention to properly utilize the full set of test data were addressed in this work. Information on the actual source content measured with a rotating rake became available and subsequent testing was performed to reduce the uncertainty in the liner impedances. Utilization of the rotating rake information to specify the incident sound field illustrated source effects and demonstrated the applicability of the statistical fan source model used in the liner design process. Testing of the centerbody material demonstrated the rigid wall assumption to be appropriate. In contrast to the initial estimate, the addition of the coarse screen increased resistance by only $0.036 \rho c$ (with negligible impact on the reactance). Comparisons of updated predicted attenuations with measured values were greatly improved, providing further confidence in the conclusions drawn and in continuing investigations to the farfield data.

In addition to bringing these areas of interest to light, this study provided further evidence to support the expectation that the CDL code can be used to investigate various liner configurations and provide conservative attenuation predictions that correctly capture measured trends. It has also provided an excellent opportunity to improve the integrated use of duct acoustic propagation/radiation and liner modeling tools for broadband liner design.

\section{Acknowledgments}

The authors wish to thank Martha Brown, Carol Harrison, and Brian Howerton for their assistance in determining the contribution of the coarse screen to the liner assembly impedance. The authors would also like to acknowledge the efforts of D. Podboy, L. Smith, R. Loew, B. Groening, and E. Mysliwiec of TFOME for their support in model assembly and testing. The contributions of Earl Ayle, Fumitaka Ichihashi, and Clark Smith of Hexcel Corporation were also key to the liner core development.

This research was funded by the Advanced Air Transport Technology Project of NASA's Advanced Air Vehicles Program.

\footnotetext{
${ }^{\mathrm{b}}$ The associated reduction in confidence interval size is likely due to an increase in the number of cut-on modes, as all are included in the sound source.
} 


\section{References}

${ }^{1}$ Nark, D. M., Jones, M. G., Sutliff, D. L., Ayle, E., and Ichihashi, F., "Improved Broadband Liner Optimization Applied to the Advanced Noise Control Fan," AIAA Paper 2014-3103, 2014.

${ }^{2}$ Sutliff, D. L., Jones, M. G., and Nark, D. M., "In-Duct and Far-field Experimental Measurements from the ANCF for the Purpose of Improved Broadband Liner Optimization," AIAA Paper 2014-3231, 2014.

${ }^{3}$ Loew, R. A., Lauer, J. T., McAllister, J., and Sutliff, D. L., "The Advanced Noise Control Fan,” NASA TM 2006-214368, 2006, (Also AIAA 2006-3150).

${ }^{4}$ McAllister, J., Loew, R. A., Lauer, J. T., and Sutliff, D. L., "The Advanced Noise Control Fan Baseline Measurements," NASA TM 2009215595, 2009, (Also AIAA 2009-0624).

${ }^{5}$ Sutliff, D. L., "A Mode Propagation Database Suitable for Code Validation Utilizing the NASA Glenn Advanced Noise Control Fan and Artificial Sources," AIAA Paper 2014-0719, 2014.

${ }^{6}$ Sutliff, D. L., “Turbofan Duct Mode Measurements Using a Continuously Rotating Microphone Rake," International Journal of Aeroacoustics, Vol. 6, No. 2, 2007, pp. 147-170.

${ }^{7}$ Nark, D. M., Farassat, F., Pope, D. S., and Vatsa, V., "The Development of the Ducted Fan Noise Propagation and Radiation Code CDUCTLaRC," AIAA Paper 2003-3242, 2003.

${ }^{8}$ Dougherty, R. P., “A Wave-Splitting Technique for Nacelle Acoustic Propagation,” AIAA Paper 97-1652, 1997.

${ }^{9}$ Dougherty, R. P., “A Parabolic Approximation for Flow Effects on Sound Propagation in Nonuniform, Softwall, Ducts," AIAA Paper 99$1822,1999$.

${ }^{10}$ Lan, J. H., “Turbofan Duct Propagation Model,” NASA CR 2001-211245, 2001.

${ }^{11}$ Zlavog, G. and Eversman, W., "Source effects on attenuation in lined ducts. Part I: A statistically based computational approach," Journal of Sound and Vibration, Vol. 307, No. 1-2, 2007, pp. 113-138.

${ }^{12}$ Zlavog, G. and Eversman, W., "Source effects on attenuation in lined ducts. Part II: Statistical properties," Journal of Sound and Vibration, Vol. 307, No. 1-2, 2007, pp. 139-151.

${ }^{13}$ Nark, D. M., “Assessment of Radiated Fan Noise Prediction Capabilities using Static Engine Test Data," AIAA Paper 2011-2807, 2011.

${ }^{14} \mathrm{Kraft}, \mathrm{R}$. E., Theory and Measurement of Acoustic Wave Propagation in Multi-Segmented Rectangular Flow Ducts, Ph.D. thesis, University of Cincinnati, 1976.

${ }^{15}$ Rice, E. J., "Modal Propagation Angles in Ducts with Soft Wall and Their Connection with Suppressor Performance," AIAA Paper 79-0624, 1979.

${ }^{16}$ Zlavog, G. and Eversman, W., “Source Effects on Realized Attenuation in Lined Ducts,” AIAA Paper 2003-3247, 2003.

\begin{tabular}{|l|c|c|c|}
\cline { 2 - 4 } \multicolumn{1}{c|}{} & Axial LE & Axial TE & Circumferential Extent \\
\hline Section 1 & 0 in & 7 in $(17.78 \mathrm{~cm})$ & $0^{\circ}<\theta<360^{\circ}$ \\
\hline Section 2 & 7 in $(17.78 \mathrm{~cm})$ & 9 in $(22.86 \mathrm{~cm})$. & $0^{\circ}<\theta<180^{\circ}$ \\
\hline Section 3 & 7 in $(17.78 \mathrm{~cm})$ & 9 in $(22.86 \mathrm{~cm})$ & $180^{\circ}<\theta<360^{\circ}$ \\
\hline Section 4 & 9 in $(22.86 \mathrm{~cm})$ & 16 in $(40.64 \mathrm{~cm})$ & $0^{\circ}<\theta<360^{\circ}$ \\
\hline
\end{tabular}

Table 1: Liner segment dimensions 


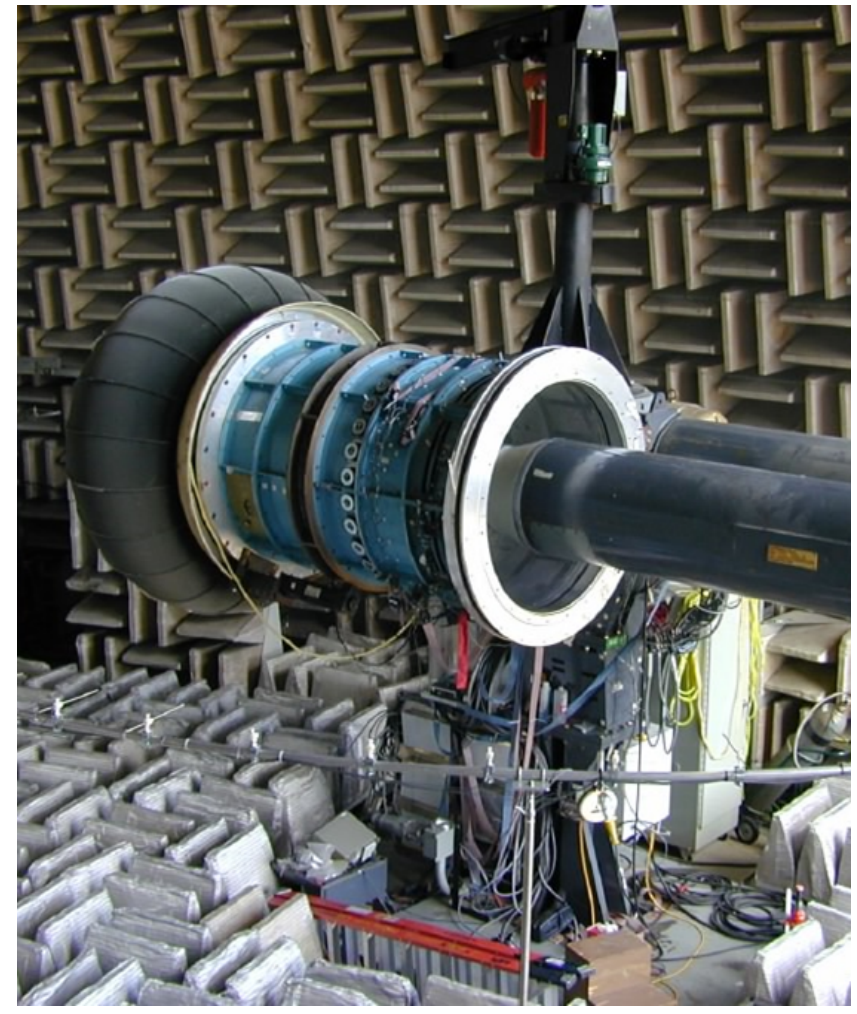

(a) Standard ANCF configuration.

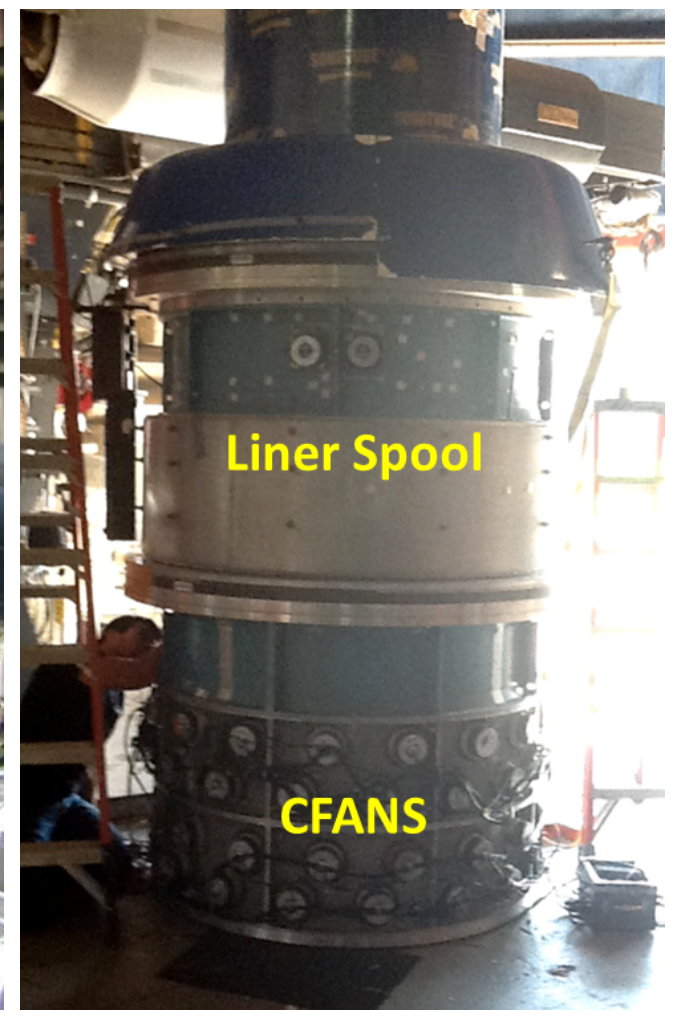

(b) Stack-up in vertical, off-stanchion orientation (No Flow).

Figure 1: Advanced Noise Control Fan (ANCF) rig configurations.

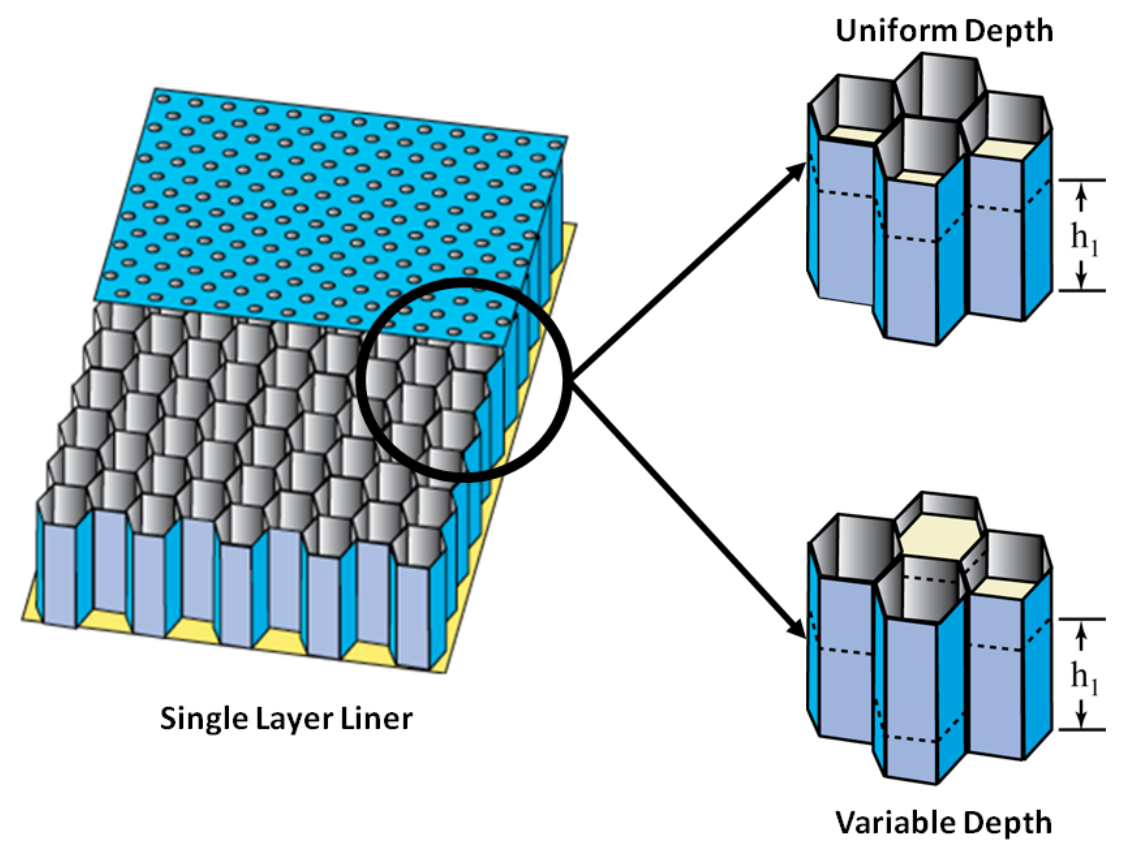

Figure 2: Parent single layer liner and resultant two-layer liner configurations (uniform and variable depth) via meshcap insertion. 


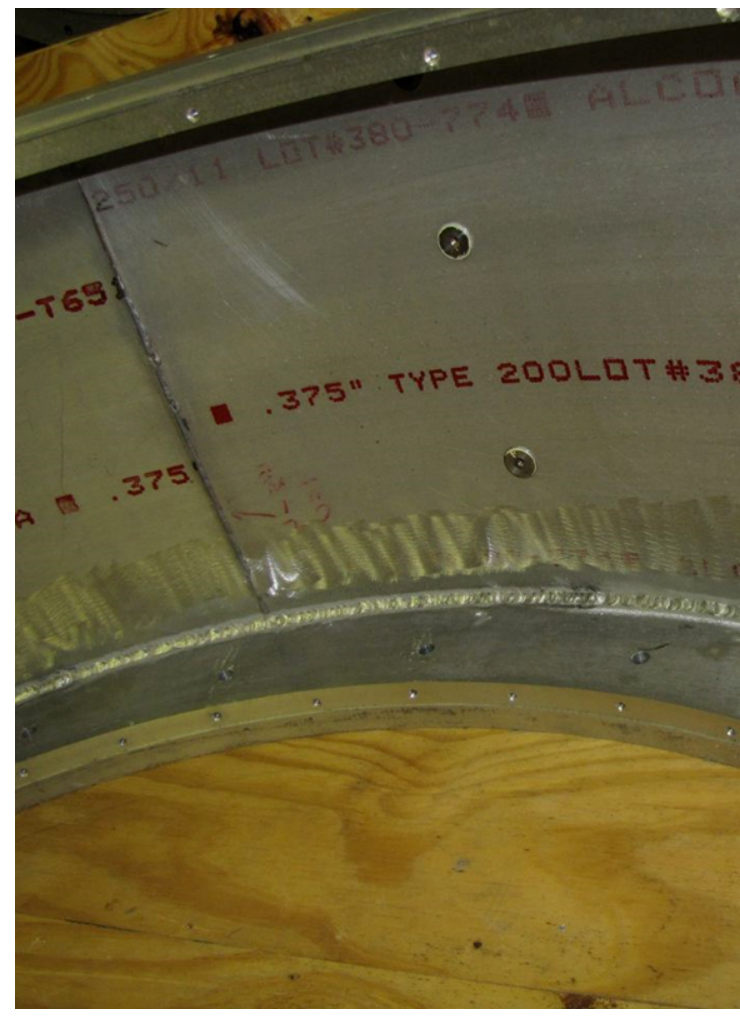

(a) Liner spool piece with empty cavity.

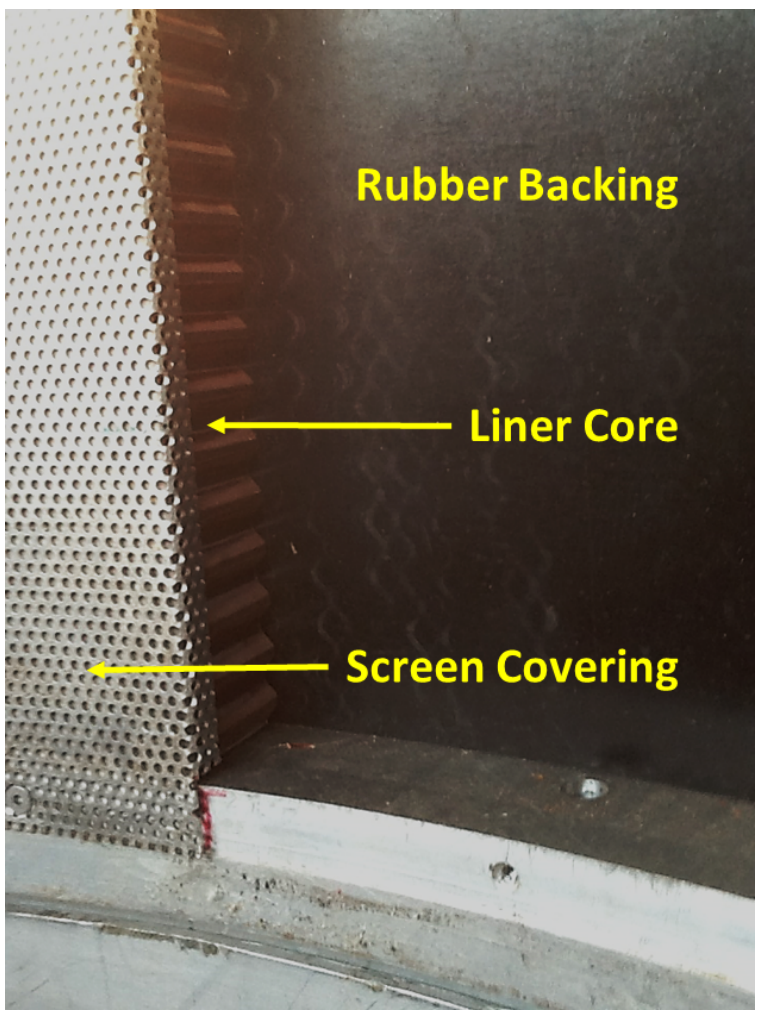

(b) Complete liner spool build-up.

Figure 3: Photographs of the liner assembly process.

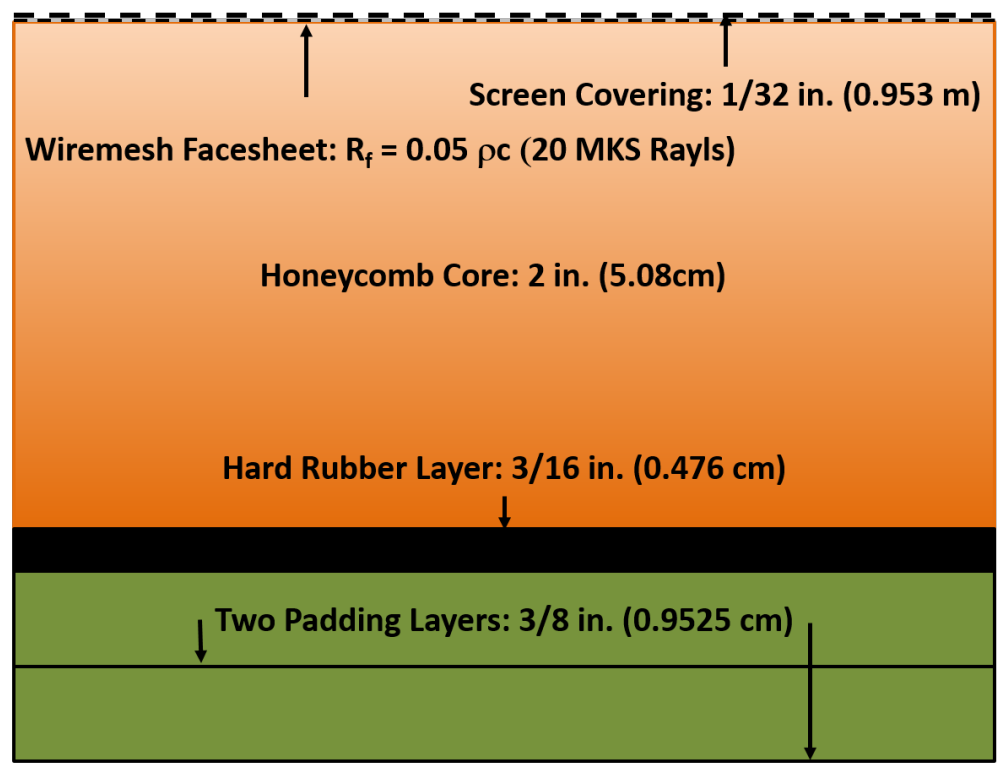

Figure 4: Cross-section of liner assembly. 


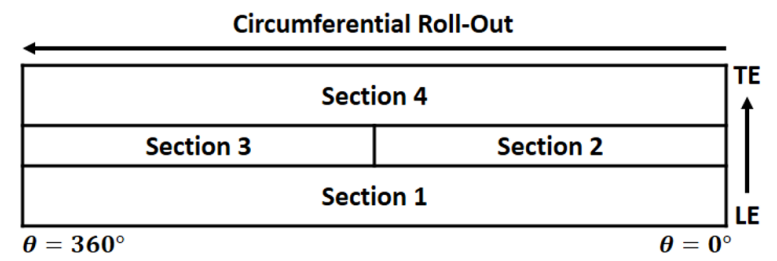

(a) Liner Segments

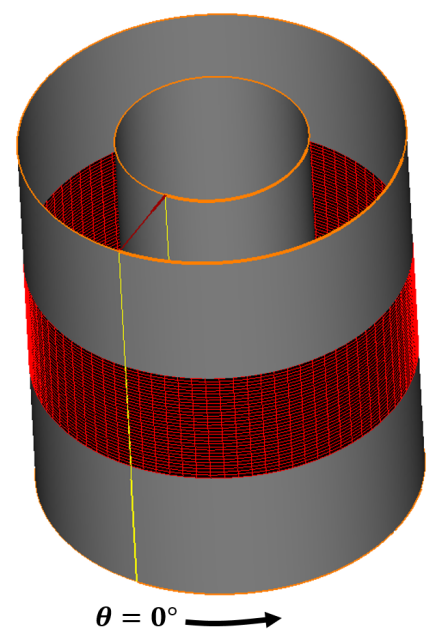

(b) Configuration B: Fully exposed liner.

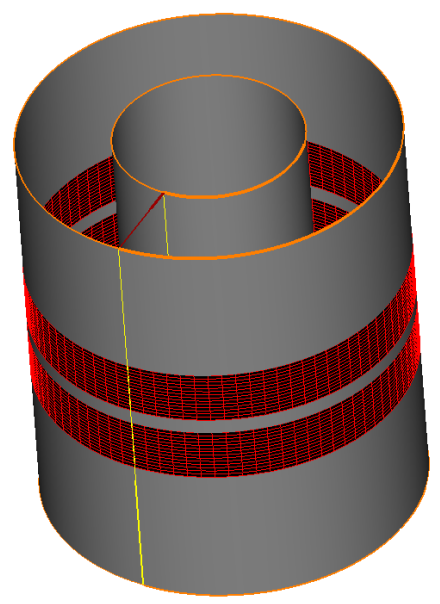

(d) Configuration D: $360^{\circ}$ hardwall splice.

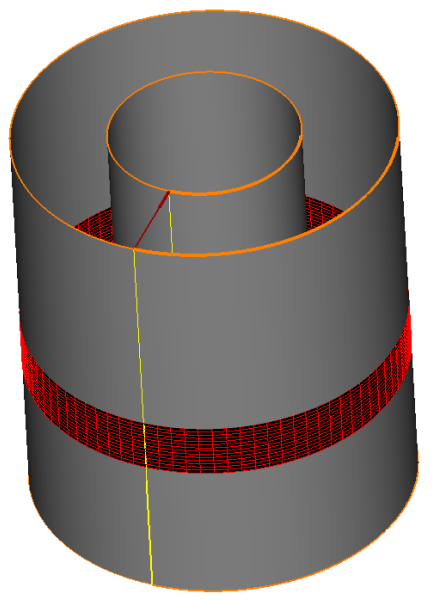

(c) Configuration C: Partially exposed liner.

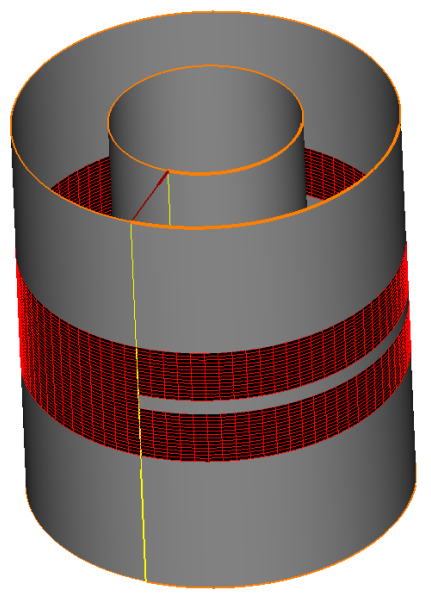

(e) Configuration E: $180^{\circ}$ hardwall splice.

Figure 5: Liner configurations. 


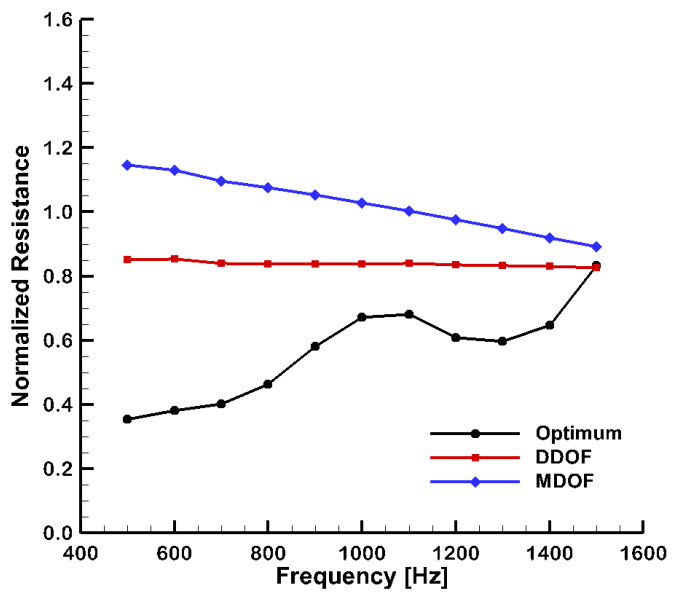

(a) Normalized Resistance.

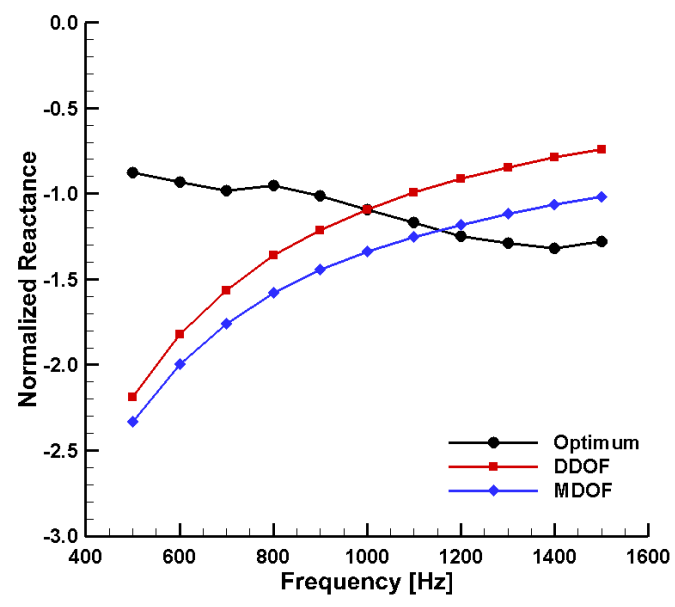

(b) Normalized Reactance.

Figure 6: Comparison of optimum and design impedance spectra for the 24 inch diameter centerbody $(\tau=0.5)$.

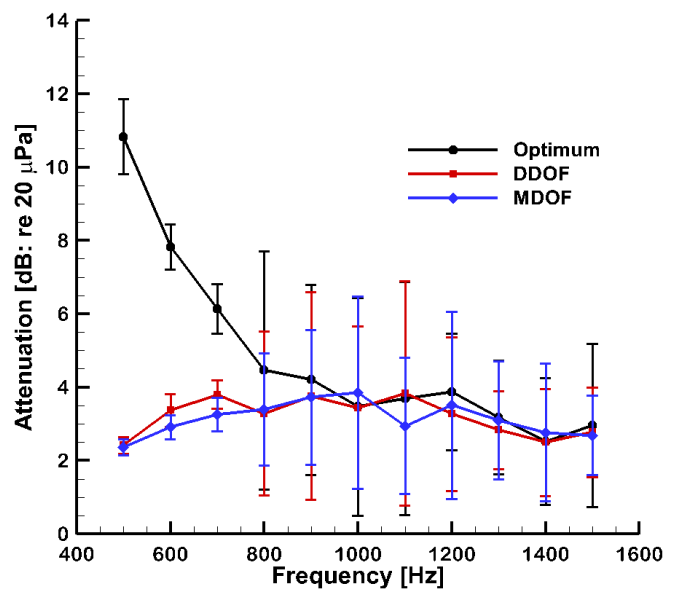

(a) $\tau=0.5(L / H=0.7)$.

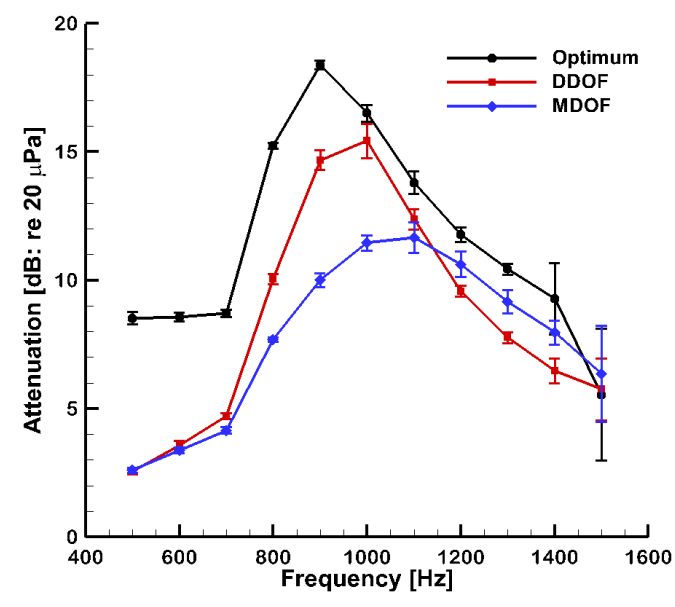

(b) $\tau=0.75(L / H=1.3)$.

Figure 7: Comparison of predicted attenuation values for the two centerbody configurations using statistical source input (random amplitude and phase for all cut-on modes). Error bars indicate 95\% confidence intervals on the predicted attenuation values. 


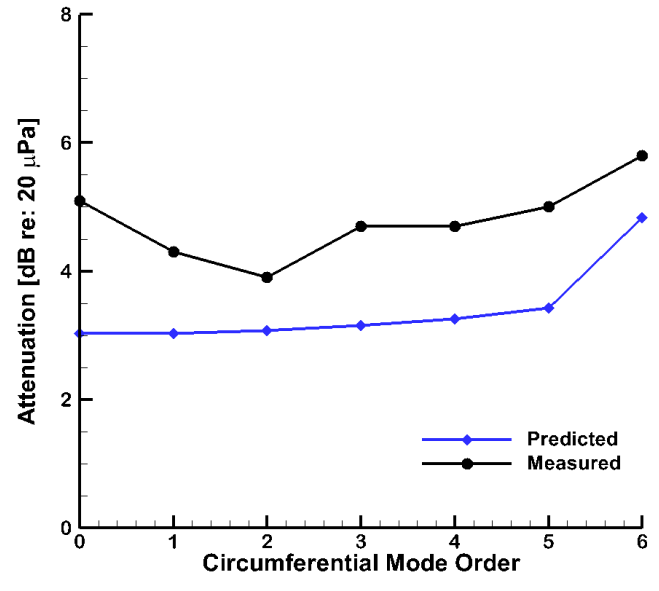

(a) Configuration C: $\tau=0.75, f=1100 \mathrm{~Hz}$.

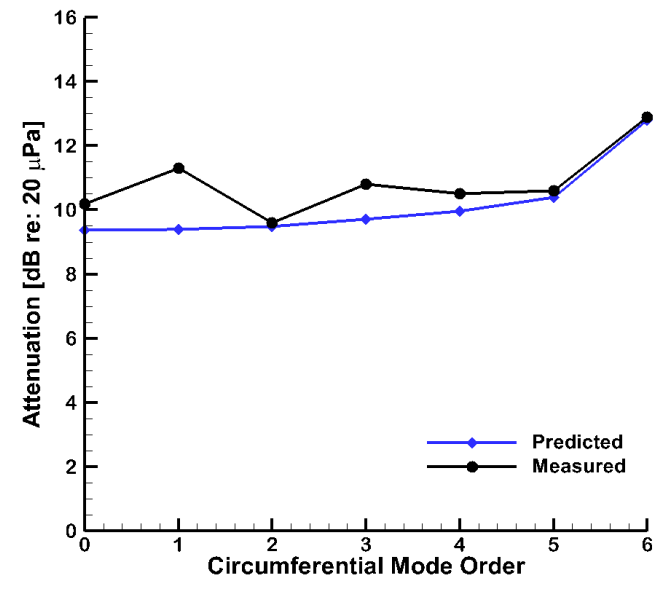

(b) Configuration D: $\tau=0.75, f=1100 \mathrm{~Hz}$.

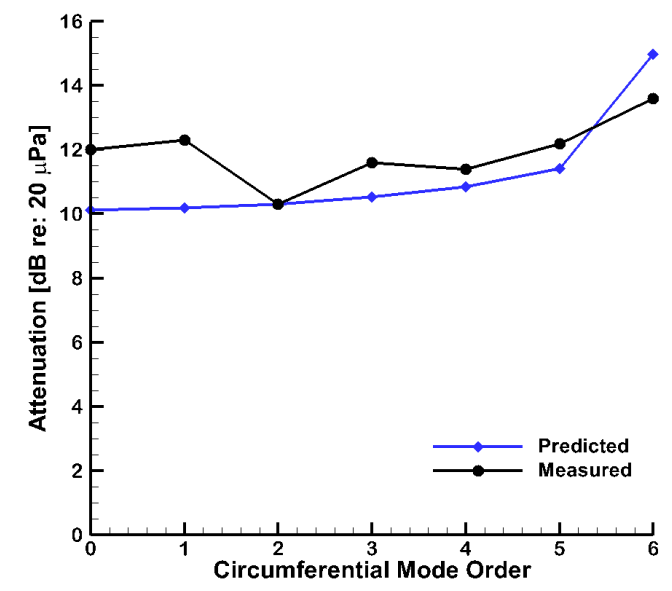

(c) Configuration E: $\tau=0.75, f=1100 \mathrm{~Hz}$.

Figure 8: Comparison of predicted and measured attenuation values for the MDOF liner with modal source input. Predicted modal source input based on rotating rake data (amplitude and phase). 


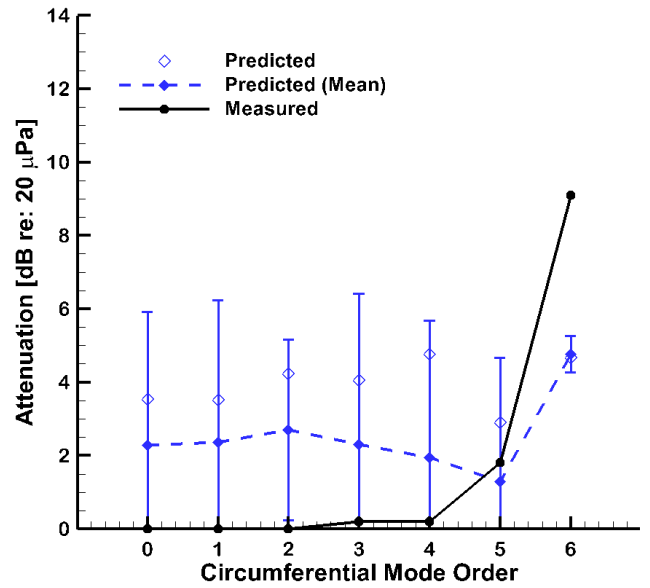

(a) Configuration C: $\tau=0.5, f=1100 \mathrm{~Hz}$.

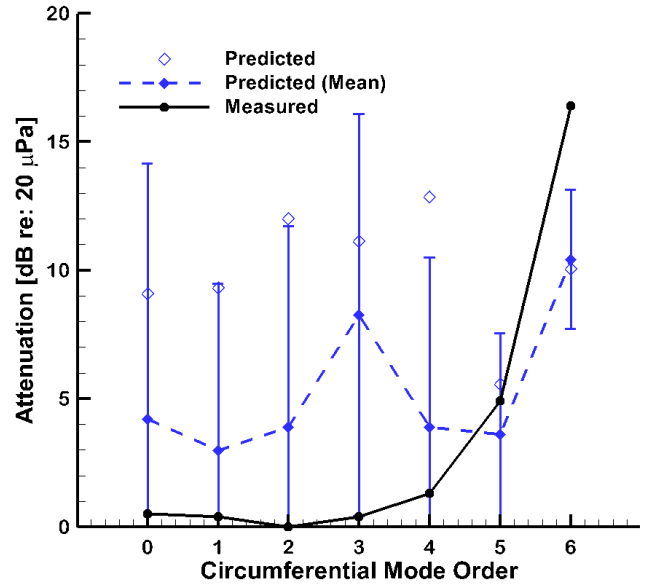

(c) Configuration E: $\tau=0.5, f=1100 \mathrm{~Hz}$.

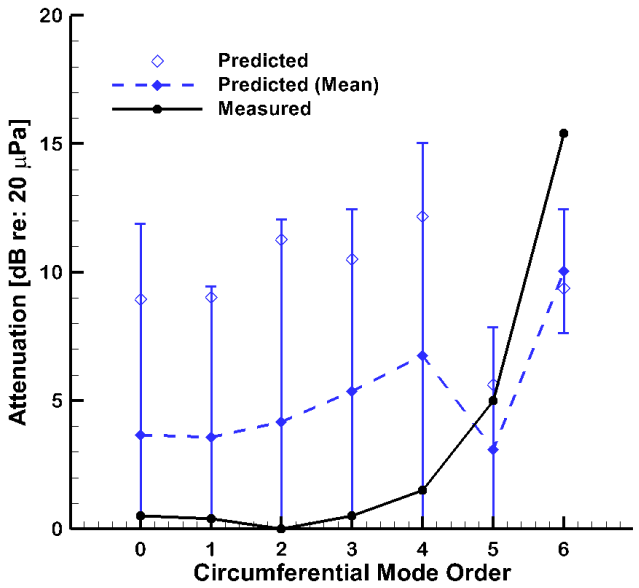

(b) Configuration D: $\tau=0.5, f=1100 \mathrm{~Hz}$.

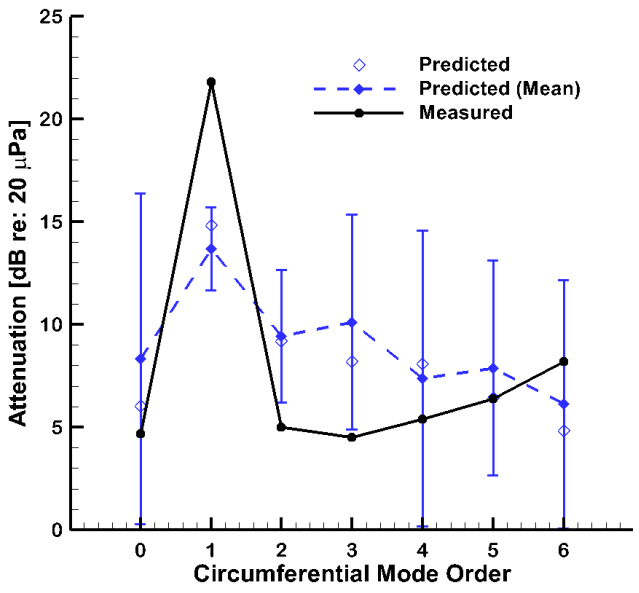

(d) Configuration E: $\tau=0.75, f=1500 \mathrm{~Hz}$.

Figure 9: Comparison of predicted and measured attenuation values for the MDOF liner with modal source input. Open diamond symbols represent predicted attenuation based on direct rotating rake data. Closed diamond symbols represent mean attenuation values based on rotating rake modal amplitudes with randomized phase. Error bars indicate estimated $95 \%$ confidence intervals associated with the predicted mean attenuation values. 


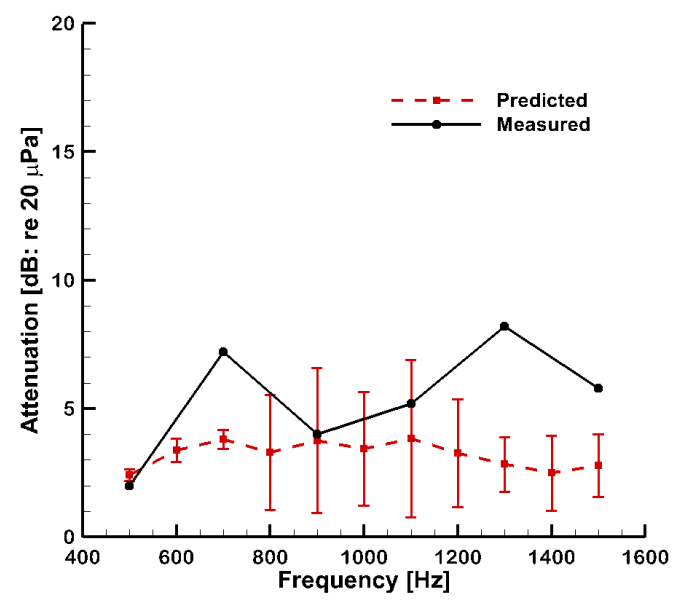

(a) Statistical source input.

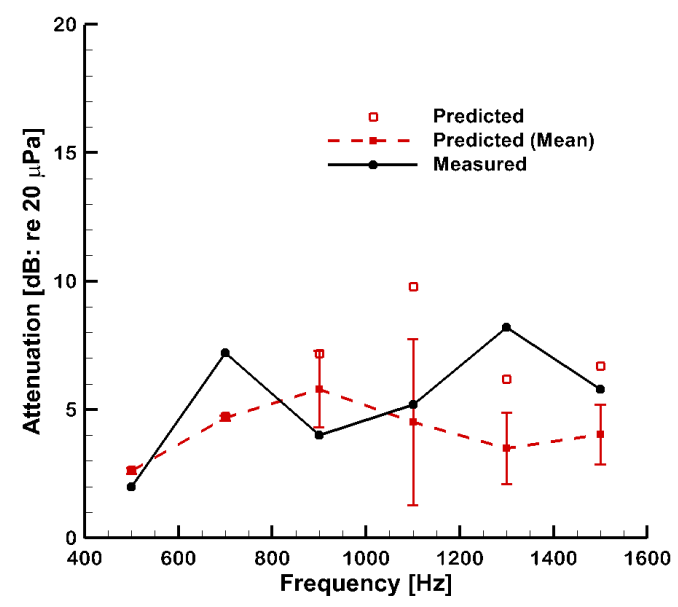

(b) Rotating rake source input.

Figure 10: Comparison of predicted and measured attenuation values for the DDOF liner in configuration B $(\tau=0.5)$ with "random" source input. (a) Random modal amplitude and phase for all cut-on modes, (b) Modal amplitudes based on rotating rake data with random phase. Error bars indicate estimated $95 \%$ confidence intervals on the predicted attenuation values.

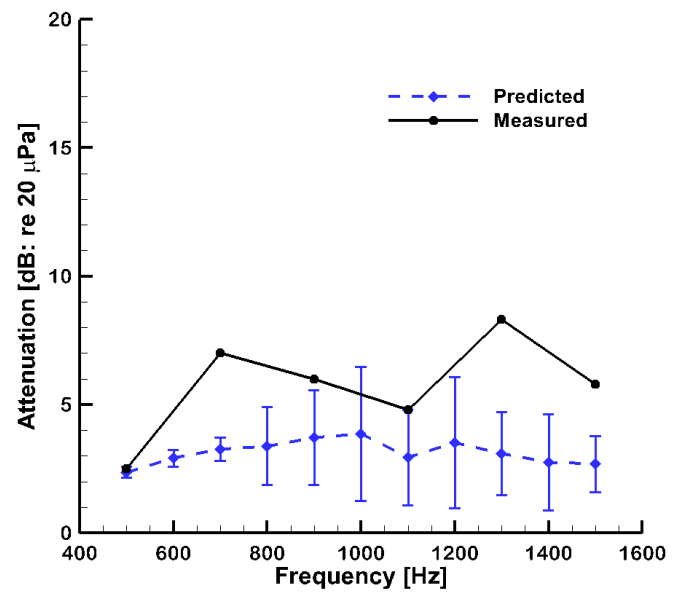

(a) Statistical source input.

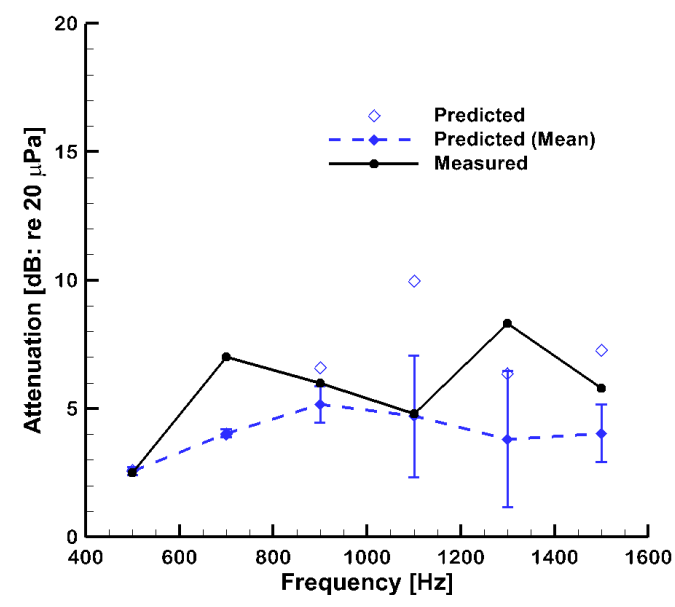

(b) Rotating rake source input.

Figure 11: Comparison of predicted and measured attenuation values for the MDOF liner in configuration B $(\tau=0.5)$ with "random" source input. (a) Random modal amplitude and phase for all cut-on modes, (b) Modal amplitudes based on rotating rake data with random phase. Error bars indicate estimated $95 \%$ confidence intervals on the predicted attenuation values. 


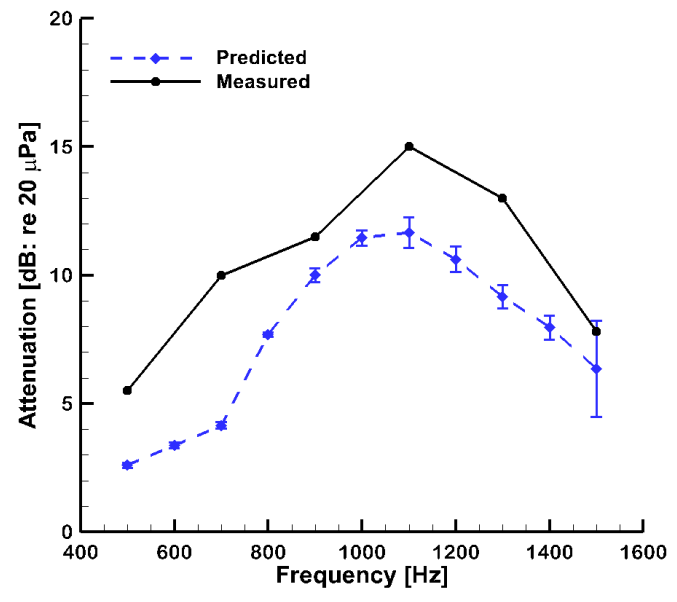

(a) Statistical source input.

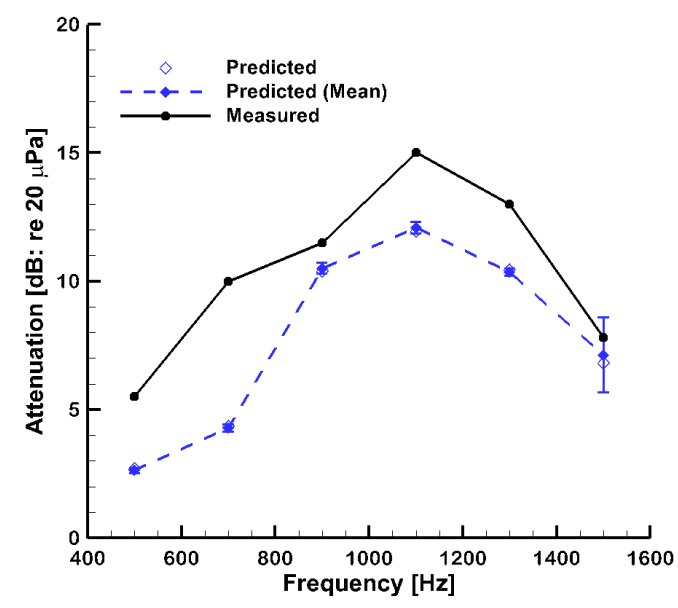

(b) Rotating rake source input.

Figure 12: Comparison of predicted and measured attenuation values for the MDOF liner in configuration $B(\tau=0.75)$ with "random" source input. (a) Random modal amplitude and phase for all cut-on modes, (b) Modal amplitudes based on rotating rake data with random phase. Error bars indicate estimated $95 \%$ confidence intervals on the predicted attenuation values. 